\title{
Practical Structural Design Approach of Multiconfiguration Planar Single-Loop Metamorphic Mechanism with a Single Actuator
}

\author{
Qiang Yang ${ }^{1^{*}}\left(\mathbb{0}\right.$, Guangbo Hao ${ }^{2}$, Shujun $\mathrm{Li}^{1,3}$, Hongguang Wang ${ }^{3}$ and Haiyang $\mathrm{Li}^{1}$
}

\begin{abstract}
As a type of multiconfiguration mechanism that can operate in an under-actuated state, metamorphic mechanisms were proposed more than two decades ago and attracted significant interest. Studies on structural synthesis of metamorphic mechanisms tend to focus more on metamorphic techniques and the structural synthesis of source mechanisms for metamorphic mechanisms. By designing different constraint architectures of metamorphic joints, multistructures can be obtained from the same source metamorphic mechanism. To determine the constraint architectures of metamorphic joints and their different assembly combinations, a kinematic status matrix and a corresponding constraint status matrix are constructed based on the metamorphic cyclogram of a source mechanism. According to the equivalent resistance gradient model and the constraint status matrix, an equivalent resistance matrix for the metamorphic joints is proposed. A structural synthesis matrix of the metamorphic mechanism is then obtained from the equivalent resistance matrix by deducing the constraint form vectors of the metamorphic joints. Furthermore, a kinematic diagram synthesis of the source metamorphic mechanism of a planar single-loop metamorphic mechanism is proposed, which is based on only the 14 one- or zero-degrees-of-freedom linkage groups. The entire structural design method of a metamorphic mechanism is based on the structural synthesis matrix and is presented as a systematic process. Finally, the proposed structural design approach is illustrated by two examples to verify its feasibility and practicality. This study provides an effective method for designing a practical multi-mobility and multiconfiguration planar single-loop metamorphic mechanism with a single actuator.
\end{abstract}

Keywords: Metamorphic mechanism, Structural design, Form of metamorphic joints, Multiconfiguration, Equivalent resistance, Single actuator

\section{Introduction}

A fundamental tenet of mechanism design is to minimize the number of actuators required to perform a given operational task. Therefore, the challenge lies in designing such mechanisms to achieve the prescribed motion output with fewer actuators [1]. Generally, under-actuated mechanisms are more cost-effective than general multimobility mechanisms with the same operational requirements. As a type of mechanism that can

\footnotetext{
${ }^{*}$ Correspondence: qiangyang@mail.neu.edu.cn

${ }^{1}$ College of Mechanical Engineering and Automation, Northeastern

University, Shenyang 110819, China

Full list of author information is available at the end of the article
}

operate in an under-actuated state, metamorphic mechanisms were proposed more than two decades ago [2] and attracted significant interest. Li et al. [3] presented a method for the structural synthesis of metamorphic mechanisms based on configuration transformations. Liu et al. [4] determined methods for self-locking by applying geometric constraints to kinematic joints. Focusing on a class of improved link-type shape-shifting modular robot, Liu et al. [5] studied a transformation technique whose feasibility was verified by experimental results. Wang et al. [6] proposed a method based on matrix calculations to achieve structural synthesis for the source mechanisms of metamorphic mechanisms. Dai and Wang presented an approach for synthesizing a metamorphic palm 
and characterized the grasp ability of a novel metamorphic robotic hand [7]. Lan and Du [8] introduced a new adjacency matrix that can be used to trace the structural changes, find all the possible configurations, and calculate the degrees of freedom (DOFs) of a metamorphic mechanism. Ding [9] studied topological and metamorphic principles with the same parts and symmetrical structure. Zhang and Ding et al. [10] proposed a comprehensive symbolic matrix for characterizing the topology of a metamorphic mechanism involving variations of links and axial orientations of the kinematic joints. Dai et al. [11] introduced an EU-elementary matrix operation to produce the configuration transformation for modeling topological changes of metamorphic mechanisms. Li et al. [12] presented an augmented adjacency matrix to describe the topological configuration of metamorphic mechanisms. Zhang et al. [13] studied the synthesis and configuration design of metamorphic mechanisms based on biological modeling and genetic evolution and analyzed constrained metamorphic techniques and geometric constraint principles [14]. Lan [15] studied the structural components and evolution of planar metamorphic mechanisms. Zhang et al. [16] researched a metamorphic kinematic chair with the capacity to change mobility, with the DOFs changing from 1 to 3. Yang and Deng et al. [17] introduced a new matrix description with respect to increasing or unchanging links in configuration transformation, and then presented a new method for the structural synthesis of metamorphic mechanisms. Gan et al. $[18,19]$ invented a reconfigurable Hooke joint, the rT joint, the direction of whose rotation axis can be altered. They then proposed two types of metamorphic parallel mechanisms assembled with these $\mathrm{rT}$ joints. Zhang et al. [20] presented a synthesis method for metamorphic mechanisms based on constraint variation. Valsamos, Moulianitis, and Aspragathos presented a method for the kinematic synthesis of structure topologies for modular metamorphic serial manipulators [21]. Yang et al. [22] proposed a practical synthesis method for metamorphic mechanisms and studied its application to broken strand repair operations on extra-high-voltage (EHV) power transmission lines. Kong et al. [23, 24] presented structural syntheses of a class of variable-DOF single-loop mechanisms and 3-4R two-DOF parallel mechanisms. Li et al. [25-27] introduced the augmented Assur group used in the structural theory of metamorphic mechanisms, which is similar to the Assur groups in general planar mechanisms. Li and Dai [28] presented an equivalent resistance gradient model for metamorphic mechanisms. From this model, the connections between the equivalent resistance, constraint characteristics, and architectures of metamorphic joints were established. Tian et al. [29] presented a configuration synthesis method for metamorphic mechanisms based on functional analyses. Based on a type of lockable spherical joint, Guo et al. [30] proposed a new 3RRIS metamorphic parallel mechanism and analyzed its four configurations. Wei and Dai [31] presented a novel approach for synthesizing metamorphic parallel mechanisms with the ability to reconfigure motion between $1 \mathrm{R} 2 \mathrm{~T}$ and $2 \mathrm{R} 1 \mathrm{~T}$, and then the Lie group was introduced to study type synthesis using the transformation configuration space for reconfigurable parallel mechanisms [32]. Kong, Gosselin, and Angeles et al. [33-35] studied the type synthesis of parallel mechanisms, which provided a meaningful reference for the synthesis study of metamorphic mechanisms. Li et al. [36, 37] studied the structural synthesis of compliant metamorphic mechanisms based on adjacency matrix operations, which can also be used in the design of new compliant metamorphic mechanisms.

At present, studies on the structural synthesis of metamorphic mechanisms are more focused on metamorphic techniques and the structural synthesis of source mechanisms for metamorphic mechanisms. An effective method to obtain the expected working stages of metamorphic mechanisms is to design different constraint forms and structures of metamorphic joints to provide the required geometric and force constraints. Several questions remain unanswered for a given source mechanism and the constraint force demands of the metamorphic working stages. For example, which constraint forms and structures of metamorphic joints should be selected? How many architectures of metamorphic mechanisms correspond to the different metamorphic joints? Based on the equivalent resistance gradient model of metamorphic mechanisms proposed in Ref. [28], this study investigates the relationship between kinematic status, resistant forces, and constraint forms and structures of metamorphic joints. A practical structural synthesis method is then presented to determine the different constraint forms and structures of constrained metamorphic joints. Finally, the combinations are assembled to obtain structures for a multi-DOF planar metamorphic mechanism with a single actuator to satisfy the functional and operational requirements for a practical working task. The remainder of this paper is organized as follows: Section 2 introduces the kinematic and constraint status matrix of metamorphic joints based on the metamorphic cyclogram of the metamorphic mechanism. Section 3 reviews the equivalent resistance gradient model originally presented in Ref. [28] and an equivalent resistance matrix is presented. In Section 4, several typical constrained structures for metamorphic joints are given, and a structural synthesis matrix of the metamorphic mechanism is proposed. A kinematic diagram synthesis of source metamorphic mechanisms based on linkage groups is studied, 
and a systematic structural design approach of metamorphic mechanisms considering the forms and structures of metamorphic joints is presented in Section 5. Finally, Section 6 provides two examples to verify the feasibility and practicality of the proposed design approach. Finally, a discussion is presented in Section 7 and Section 8 concludes the paper.

\section{Kinematic and Constraint Status Matrix of Metamorphic Mechanisms}

A general metamorphic process includes configuration transformation, configuration keeping, and working sequence problems. A typical constrained metamorphic process for a metamorphic mechanism is as follows. First, the $n$-DOF source metamorphic mechanism is degenerated into single-DOF mechanisms by constraining $n-1$ metamorphic joints to be static, thereby eliminating $n-1$ DOFs to form one of the working stages. The metamorphic mechanism operates in this working configuration for some part of the task. Then, one constrained metamorphic joint is released and the other metamorphic joints are rendered static to change the working configuration and form another working stage. The required working sequences are controlled by accurately constraining the different metamorphic joints. To achieve all the metamorphic working configurations, the metamorphic joints must be constrained alternately according to the required working stages and working sequences. Clearly, $n$ metamorphic joints should be arranged into an $n$-DOF source metamorphic mechanism to obtain $n$ work stages (configurations).

\subsection{Metamorphic Cyclogram of Metamorphic Mechanism}

Compared with the actuator joint and general joints, both of which operate continuously, the kinematic status of each constrained metamorphic joint transfers between moving and static depending on the changing of constraint forces to form the corresponding working stages. Figure 1 shows the working status of all joints with the actuator movement of a 3-DOF metamorphic mechanism. The displacement (rotation angle/moving distance) $\theta$ of the actuator is shown on the horizontal axis, and the kinematic status of the joints during the corresponding working stages or metamorphic configurations is shown on the vertical axis. The configuration transformation and kinematic status of metamorphic joints can be described by a metamorphic cyclogram.

\subsection{Kinematic and Constraint Status Matrix of Metamorphic Mechanism}

According to the metamorphic cyclogram shown in Figure 1, a kinematic status matrix $\boldsymbol{M}$ of metamorphic joints can be constructed:

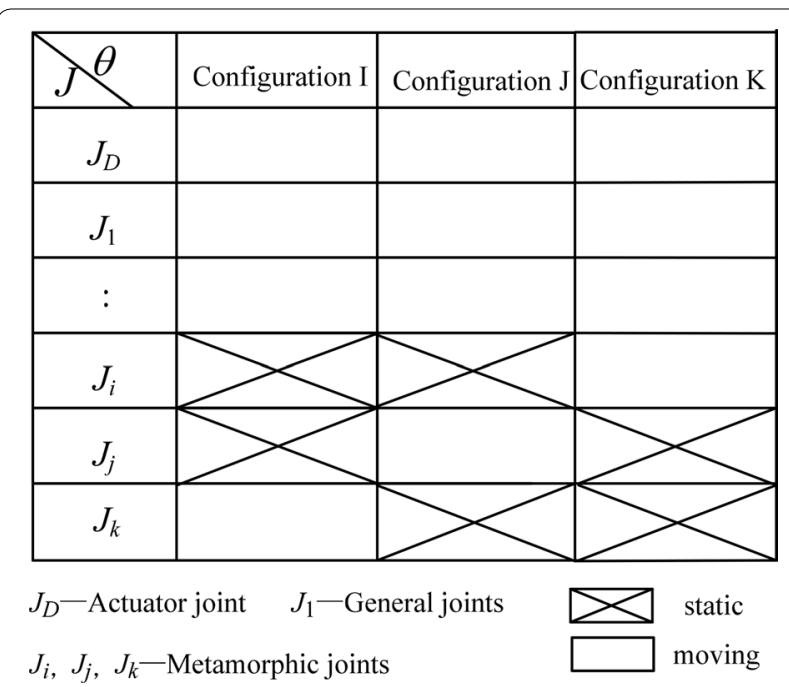

Figure 1 Metamorphic cyclogram of the 3-DOF metamorphic mechanism

$$
M=\left[\begin{array}{ccc}
0_{i i} & 0_{i j} & 1 \\
0_{j i} & 1 & 0_{j k} \\
1 & 0_{k j} & 0_{k k}
\end{array}\right]
$$

where $0_{i j}$ denotes that metamorphic joint $i$ remains static during the working stage/configuration $j$ and 1 denotes that the metamorphic joint keeps moving; the working stages/configurations are shown in the columns of the matrix, and the kinematic status of metamorphic joints in different working stages are shown in the rows.

Correspondingly, the constraint status matrix $W$ of the metamorphic joints can be obtained according to Eq. (1):

$$
\boldsymbol{W}=\left[\begin{array}{ccc}
1_{i i} & 1_{i j} & 0 \\
1_{j i} & 0 & 1_{j k} \\
0 & 1_{k j} & 1_{k k}
\end{array}\right]
$$

where $1_{i j}$ denotes that metamorphic joint $i$ remains constrained during the working stage/configuration $j$ and 0 denotes that the metamorphic joint remains unconstrained; the working stages/configurations are shown in the columns of matrix, and the constraint status of metamorphic joints in different working stages is shown in the rows.

\section{Equivalent Resistance Gradient Model and Equivalent Resistance Matrix}

\subsection{Equivalent Resistance Gradient Model of Metamorphic} Mechanisms

For constrained metamorphic mechanisms, only one metamorphic joint moves in a working stage while the others are static. The interchange from moving to static of a metamorphic joint is controlled by the constrained 
forces generated from the practical implementation of the metamorphic joint. In Ref. [28], the equivalent resistance coefficient is proposed to describe the relationship between the working/kinematic status and constraint resistance characteristics. This concept also helps to compare the constrained forces between revolute and prismatic metamorphic joints. It is defined as the ratio of the force and/or torque in the moving direction provided by the constraint of the metamorphic joint to the force and/or torque in the moving direction acting on the metamorphic joint in the working process.

$$
f_{e}\left(\theta_{i}\right)=\frac{\boldsymbol{F}_{\mathrm{c}}\left(\theta_{i}\right)}{\boldsymbol{F}\left(\theta_{i}\right)}=\frac{\boldsymbol{T}_{\mathrm{c}}\left(\theta_{i}\right)}{\boldsymbol{T}\left(\theta_{i}\right)},(i=1,2, \ldots, m),
$$

where $f_{e}\left(\theta_{i}\right)$ is the equivalent resistance coefficient of the metamorphic joint, $\theta_{i}$ is the displacement of the actuator, and $m$ is the number of working stages. $\boldsymbol{F}_{\mathrm{c}}\left(\theta_{i}\right)$ and $\boldsymbol{T}_{\mathrm{c}}\left(\theta_{i}\right)$ are the resistant force and resistance torque in the moving direction provided by the constraint of the metamorphic joint, respectively. $\boldsymbol{F}\left(\theta_{i}\right)$ and $\boldsymbol{T}\left(\theta_{i}\right)$ are the actual force and torque in the moving direction acting on the metamorphic joint in the working process, respectively. To show the resistance characteristics of the metamorphic joint and then obtain the constraint form and structure information of the metamorphic joints, Eq. (3) can be modified as:

$$
\begin{gathered}
f_{e m}\left(\theta_{i}\right)=\frac{\boldsymbol{F}_{\mathrm{c}}\left(\theta_{i}\right)}{\boldsymbol{F}_{\text {min }}}=\frac{\boldsymbol{T}_{\mathrm{c}}\left(\theta_{i}\right)}{\boldsymbol{T}_{\text {min }}}, \\
f_{e s}\left(\theta_{i}\right)=\frac{\boldsymbol{F}_{\mathrm{c}}\left(\theta_{i}\right)}{\boldsymbol{F}_{\max }}=\frac{\boldsymbol{T}_{\mathrm{c}}\left(\theta_{i}\right)}{\boldsymbol{T}_{\max }},
\end{gathered}
$$

where $f_{e m}\left(\theta_{i}\right)$ is the equivalent resistance coefficient of the moving metamorphic joint in the working stages. $\boldsymbol{F}_{\text {min }}$ and $\boldsymbol{T}_{\min }$ are the minimum actual force and torque acting on the moving metamorphic joint in the working stages, respectively. $f_{e s}\left(\theta_{i}\right)$ is the equivalent resistance coefficient of the static metamorphic joint in the working stages. $\boldsymbol{F}_{\max }$ and $\boldsymbol{T}_{\max }$ are the maximum actual force and torque acting on the static metamorphic joint in the corresponding working stages, respectively. $f_{e m}\left(\theta_{i}\right)$ and $f_{e s}\left(\theta_{i}\right)$ become proportional to $\boldsymbol{F}_{\mathrm{c}}\left(\theta_{i}\right)$ and/or $\boldsymbol{T}_{\mathrm{c}}\left(\theta_{i}\right)$ by modified Eqs. (4) and (5), and thus, the equivalent resistance force sketches of the metamorphic joint are similar to the force characteristic sketches of the metamorphic joint.

The moving sequences of metamorphic joints should be proportional to the equivalent resistant forces according to the law of minimum resistance of kinematics, and the equivalent resistance gradient of the metamorphic joints in the working stages of the constrained metamorphic process should be

$$
f_{e m}\left(\theta_{i}\right) \leq 1 \text { and } f_{e s}\left(\theta_{i}\right) \geq 1 .
$$

In the corresponding working stages, the equivalent resistance coefficient of the moving metamorphic joint is smaller than that of the static metamorphic joint, and Eq. (6) is called the equivalent resistance gradient model. The equivalent resistance gradient sketch of a 3-DOF metamorphic mechanism, for example, can be formed as shown in Figure 2 according to Eq. (6). The sketch of a 3-DOF metamorphic mechanism in Figure 2 describes the change in the equivalent resistance coefficients of the three metamorphic joints in three working stages.

According to Eq. (5), when $f_{e}\left(\theta_{i}\right) \leq 1$, the metamorphic joint continues moving, and $f_{e}\left(\theta_{i}\right) \geq 1$. indicates that it remains static. As shown in Figure 2, the displacement (rotation angle/moving distance) $\theta$ of the actuator is shown on the horizontal axis, and the equivalent resistance coefficients during the corresponding working stages/metamorphic configurations are shown on the vertical axis. In configuration I, the metamorphic joint $i$ continues moving and the other two remain static. In configuration II, joint $j$ keeps moving, and joint $k$ keeps moving in configuration III. Undoubtedly, the true shape of each curve depends on the actual force acting on each metamorphic joint and the practical structures.

\subsection{Equivalent Resistance Matrix of Metamorphic Mechanisms}

Comparing Eq. (6) to Figure 2, the equivalent resistance matrix of a 3-DOF metamorphic mechanism is

$$
\boldsymbol{F}=\left(\begin{array}{ccc}
0_{i 1} & f_{i 2} & f_{i 3} \\
f_{j 1} & 0_{j 2} & f_{j 3} \\
f_{k 1} & f_{k 2} & 0_{k 3}
\end{array}\right),
$$

where $f_{i j}>1$ denotes metamorphic joint $i$ being constrained in working stage $j$ and $0_{i j}$ denotes metamorphic joint $i$ being unconstrained in stage $j$. Working stages are shown in the columns of the matrix, and the constraint status is shown in the rows.

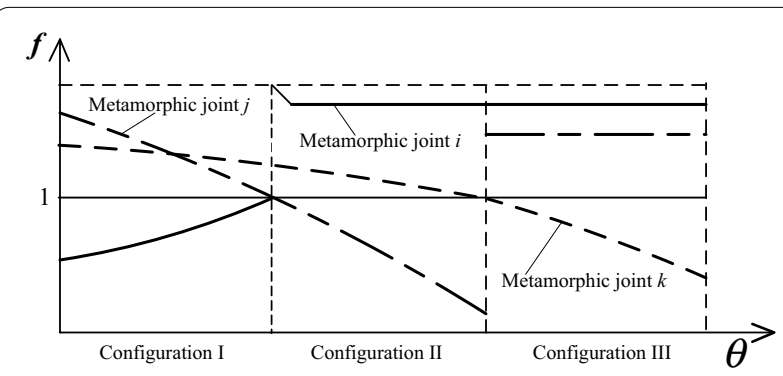

Figure 2 Equivalent resistance gradient sketch of a 3-DOF metamorphic mechanism 


\section{Structural Synthesis Matrix of Metamorphic Mechanism}

To obtain the motions of the metamorphic joints that are described by the metamorphic cyclogram, the resistance force of each constrained metamorphic joint is first calculated, and then a constrained form/structure that can achieve the corresponding resistance force is designed.

\subsection{Constraint Forms/Structures and Force Characteristic Sketches of Metamorphic Joints}

There are several ways to obtain constrained metamorphic joints. For example, constraints may be generated by structures that include geometric constraints, geometric limitations, and force constraints (e.g., spring force, gravity, reaction force). Another method is to implement constraints on the kinematic geometry arrangements (e.g., singularity position). Some typical forms for constrained metamorphic joints include the following: (1) Geometric constraint: geometric limitation, or lock/unlock the limit controlled by profiles of links and/or joints (e.g., cam). The geometric constraint could theoretically provide infinite constraint force at the limit position. (2) Force constraint: spring force constraint or geometric constraint controlled by spring, which provides limited constraint force. The magnitude of the force is determined by the spring force and practical structure, which are easily adjusted. (3) Combined constraint: Combined arrangements of geometric and force constraints.

Several typical constrained structures of metamorphic joints are shown in Figure 3. In Figure 3(a), both revolute and prismatic joints can have a geometric constraint that combines the two links into one at the limit position and then provides an infinite constraint force. Figure 3(b) shows revolute and prismatic joints with a geometric constraint controlled by a spring that provides a limited or adjustable constraint force at that point. Figure 3(c) shows revolute and prismatic joints constrained by spring forces. Figure 3(d) shows revolute and prismatic joints constrained by geometric and spring force constraints, respectively, during different stages of motion. Finally, revolute and prismatic joints with geometric constraint and geometric constraint controlled by spring force, respectively, are shown in Figure 3(e). Figure 3 also shows the force characteristic sketches and constraint vectors of the corresponding metamorphic joints. In the constrained vectors, 0 denotes that metamorphic joint $R_{i j}$ is moving and 1 denotes that $R_{i j}$ is static.

According to the constrained structures, constraint vectors, and force characteristic sketches of the metamorphic joints, as shown in Figure 3, the constrained forms/structures of metamorphic joint $k$ during $m$

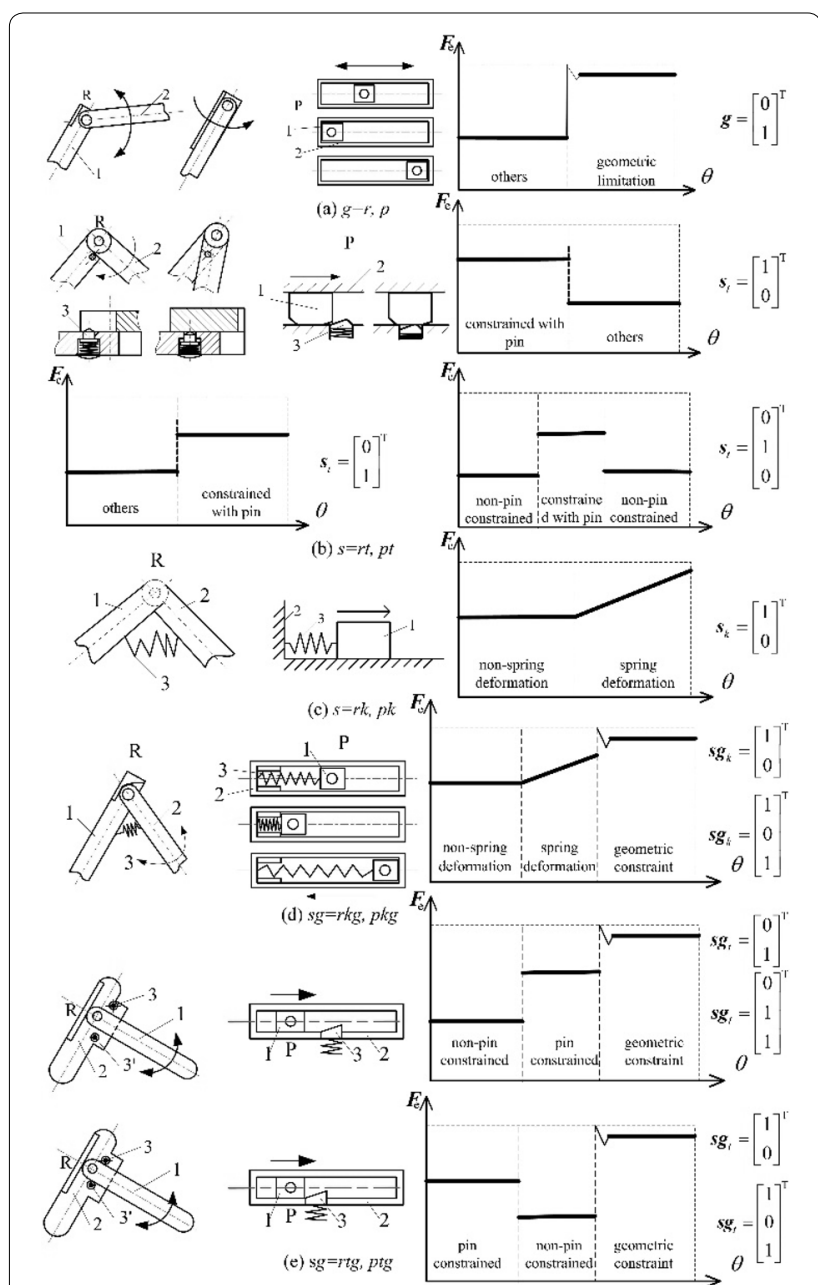

Figure 3 Constrained structures and force characteristic sketches of metamorphic joints (This figure is reprinted with permission from Ref. [26], Copyright (2015) Elsevier)

working stages can be represented by vectors for metamorphic mechanisms with a single actuator. For example,

$$
\begin{array}{r}
J_{k}=\left(\begin{array}{llllllll}
c_{1} & c_{2} & \cdots & c_{k-1} & 0_{k} & c_{k+1} & \cdots & c_{m}
\end{array}\right), \\
k=1,2, \ldots, m
\end{array}
$$

where $J$ denotes the metamorphic joint $(J=R$ for revolute joint, $J=P$ for prismatic joint), and $c_{k}$ denotes constrained forms (geometric constraint, force constraint, etc.). As shown in Figure 3, $c_{k}=g$ denotes metamorphic joints with geometric constraints, and $g=r, p$, which denote revolving or prismatic metamorphic joints with geometric constraints. $c_{k}=s$ denotes metamorphic joints with force constraints, and $s=r k, r t, p k, p t$, where $s_{t}=r t, p t$ denote revolving or prismatic metamorphic joints with geometric constraints controlled by a spring, respectively, and $s_{k}=r k, p k$ denote revolving or prismatic metamorphic joints with a spring force constraint, 
respectively. $c_{k}=s g$ denotes metamorphic joints with a spring force constraint and geometric constraint, respectively, and $s g=r k g, r t g, p k g$, ptg, which denote metamorphic joints (revolving or prismatic) with spring a force constraint and geometric constraint $\left(s g_{k}=r k g, p k g\right)$, and with a geometric constraint and geometric constraint controlled by a spring $\left(s g_{t}=r t g, p t g\right)$.

According to Figure 3, constrained forms/structures of metamorphic joints with geometric constraints or a geometric constraint controlled by a spring can be expressed as follows:

$$
\boldsymbol{J}_{k}=\left(\begin{array}{llllllll}
0_{1} & g_{2} & \cdots & g_{k-1} & g_{k} & g_{k+1} & \cdots & g_{m}
\end{array}\right) .
$$

Constrained forms/structures of metamorphic joints with force constraints can be written as

$$
\boldsymbol{J}_{k}=\left(\begin{array}{llllllll}
s_{1} & s_{2} & \cdots & s_{k-1} & 0_{k} & s_{k+1} & \cdots & s_{m}
\end{array}\right) .
$$

The constrained forms/structures of metamorphic joints with a spring force constraint and geometric constraint/geometric constraint controlled by a spring, respectively, can be expressed as

$$
\boldsymbol{J}_{k}=\left(\begin{array}{llllllll}
s_{1} & s_{2} & \cdots & s_{k-1} & 0_{k} & g_{k+1} & \cdots & g_{m}
\end{array}\right) .
$$

\subsection{Structural Synthesis Matrix of Metamorphic Mechanism}

The constrained forms/structures of metamorphic joints should correspond with an equivalent resistance sketch. According to the equivalent resistance matrix described in Eq. (7) and the definition of constrained forms/structures of metamorphic joints in Eq. (8), a structural synthesis matrix $\boldsymbol{C}$ for a constrained metamorphic mechanism is deduced to express the relationship between the constrained forms/structures of metamorphic joints and the equivalent resistance matrix $\boldsymbol{F}$ in each working stage:

$$
\boldsymbol{C}=\left(\begin{array}{c}
\boldsymbol{J}_{1} \\
\boldsymbol{J}_{2} \\
\vdots \\
\boldsymbol{J}_{k} \\
\vdots \\
\boldsymbol{J}_{m}
\end{array}\right)=\left(\begin{array}{cccccc}
0_{11} & \cdots & c_{1 i} & c_{1 j} & \cdots & c_{1 m} \\
\vdots & & \vdots & \vdots & & \vdots \\
c_{i 1} & \cdots & 0_{i i} & c_{i j} & \cdots & c_{i m} \\
c_{j 1} & \cdots & c_{i j} & 0_{j j} & \cdots & c_{j m} \\
\vdots & & \vdots & \vdots & & \vdots \\
c_{m 1} & \cdots & c_{m i} & c_{m j} & \cdots & 0_{m m}
\end{array}\right)
$$

where the constrained status of the metamorphic joints with different working stages is shown in the rows of $C$, that is, $J_{k}$ denotes constrained forms/structures of metamorphic joints $k$. The corresponding $m$ working stages are shown in the columns. $c_{i j}(i, j=1,2, \ldots, m)$ denotes the constrained forms/structures of metamorphic joint $i$ during working stage $j$.

\section{Structural Design Approach of Planar Metamorphic Mechanisms with a Single Actuator}

5.1 Kinematic Diagram Synthesis of Source Mechanism of $n$-DOF Planar Metamorphic Mechanism Based on Linkage Groups

To obtain a multi-DOF planar metamorphic mechanism operated by a single actuator, a convenient method is to make $n-1$ metamorphic joints static and eliminate $n-1$ DOFs during every working stage. This is applied to an $n$ DOF source mechanism such that

$$
\begin{gathered}
n \text {-DOF source mechanism }=(1 \text {-DOF driver })+ \\
(n-1) \times(1 \text {-DOF group })+x(0 \text {-DOF group }),
\end{gathered}
$$

where $x$ represents an arbitrary integer greater than or equal to zero. Accordingly, 1-DOF linkage groups are summarized first, and then, $n-1$ of these 1-DOF groups and arbitrarily 0-DOF groups are connected to the actuator, frame, or ahead groups in sequence, which comprise one of the $n$-DOF source mechanisms as shown in Eq. (13).

For an $n$-DOF source mechanism from the structural composition principle described in Eq. (13), we must arrange $n$ metamorphic joints in it. Therefore, select one metamorphic joint from every $n-1$ 1-DOF groups, i.e., $n-1$ metamorphic joints are determined, and then distribute the last metamorphic joint into any one of $n-11$-DOF groups or $x$ 0-DOF groups arbitrarily. For practical applications, in the $n$ metamorphic joints of the $n$-DOF source mechanism, only one metamorphic joint keeps moving in each working stage while the others remain static. In this way, an $n$-DOF planar metamorphic mechanism with a single actuator is achieved.

According to the DOF formula for planar mechanisms, $F=3 n-2 p$, where $n$ denotes the number of moving links and $p_{l}$ is the number of revolute or prismatic joints. Given that $F=1$ is desired, possible pairs of $n$ and $p_{l}$ are shown in Table 1. Excluding isomorphisms and the non-1-DOF group PPPP ( $\mathrm{R}$ denotes a revolute joint and $\mathrm{P}$ denotes a prismatic joint), there are only nine forms for 1 -DOF groups consisting of three moving links and four revolute or prismatic pairs, as shown in Figure 4.

For $n=5$ and $p_{l}=7$ or other solutions with more moving links, these mechanisms all have fewer applications compared to the 1-DOF groups in Figure 4, because of their more complex structures. Therefore, the structural

Table 1 Combination of $\boldsymbol{n}$ and $p_{\text {, of 1-DOF group }}$

\begin{tabular}{llllll}
\hline $\boldsymbol{n}$ & $\mathbf{1}$ & $\mathbf{3}$ & $\mathbf{5}$ & $\mathbf{7}$ & $\boldsymbol{\cdots}$ \\
\hline$p_{l}$ & 1 & 4 & 7 & 10 & $\cdots$ \\
\hline
\end{tabular}




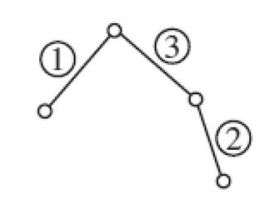<smiles>COC(O)O</smiles>

b RRRP

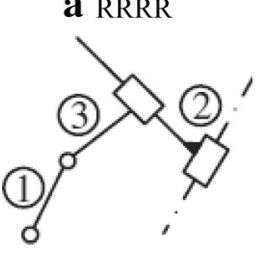<smiles>CC1CC(C)C(C(=O)O)C1O</smiles>

e RPPR

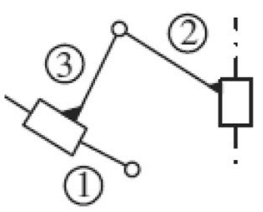

d RRPP
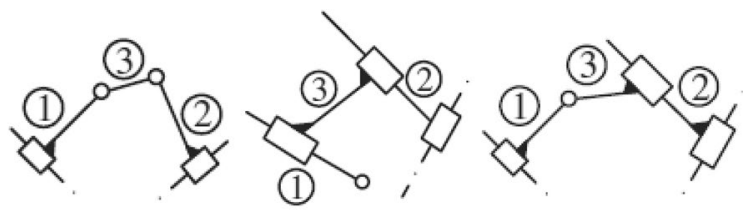

g PRRP

\section{i PRPP}

Figure 4 The whole family of 1-DOF groups consisting of $n=3$ and $p_{l}=4$

composition and synthesis based on these groups is beyond the scope of this work.

In addition to the five simplest forms of 0-DOF groups (see Figure 5), 14 basic groups could be selected to make up thousands of source mechanisms for multi-DOF metamorphic mechanisms. Furthermore, a kinematic and force calculation model based on these groups could be assembled to solve the kinematic/force analysis of all the metamorphic mechanisms composed in this way.

\subsection{Structural Design Procedure of Metamorphic Mechanism Considering Forms/Structures of Metamorphic Joints}

According to the operational requirements of a given task and the desired DOFs, the form of the output motion of the metamorphic mechanism to be designed is determined, and then a source mechanism for the metamorphic mechanism is synthesized as described in

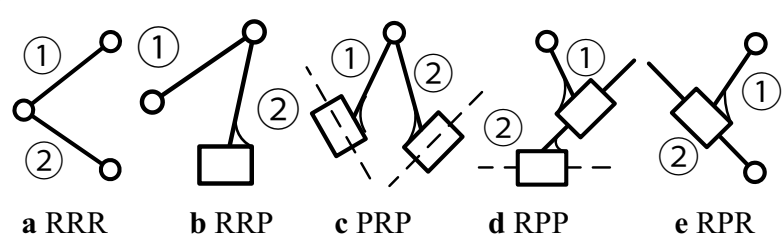

Figure 5 The whole family of 0-DOF groups consisting of $n=2$ and $p_{l}=3$
Section 5.1. After distributing the metamorphic joints and analyzing the working configurations, a metamorphic cyclogram is obtained. Therefore, the motion types $(R, P)$ of the metamorphic joints are selected, and constraint forms are determined according to the operational condition and metamorphic cyclogram. For example, suppose we have a rotational metamorphic joint $g=r, s=r k, r t$, prismatic metamorphic joint $g=p, s=p k, p t$, and revolute joint and prismatic joint with a combination constraint $s g=r k g, r t g$ or $s g=p k g$, $p t g$. Furthermore, taking the corresponding $\boldsymbol{J}_{\boldsymbol{k}}$ in Eq. (12), the structural synthesis matrix $C$ of the constrained metamorphic mechanism is obtained. Matrix $\boldsymbol{C}$ is now populated with $g, s$, and $s g$, replacing $\boldsymbol{J}_{\boldsymbol{k}}(k=1$, $2, \ldots, m)$, respectively. Corresponding with $C_{i}(i=1$, $2, \ldots, n)$, all structures of the constrained metamorphic mechanism are now designed. The structural design procedure for a multi-DOF metamorphic mechanism with a single actuator is shown in Figure 6.

1. Output motion requirements analysis, No. and types of DOF, $R$ or $P$

2. Source metamorphic mechanisms synthesis according to output motion requirements

3. Assign metamorphic joints in source mechanism and analyze working configrations

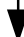

4. Kinematic/constrained status and equivalent resistance requirements analysis

\section{7}

5. Design catalogs of some typical metamorphic joints and relationship analysis between kinematic/constrained status, resistance force and structures

6. Match step 4 and 5 , obtain structural synthesis matrixes $\boldsymbol{C}$ and all structures

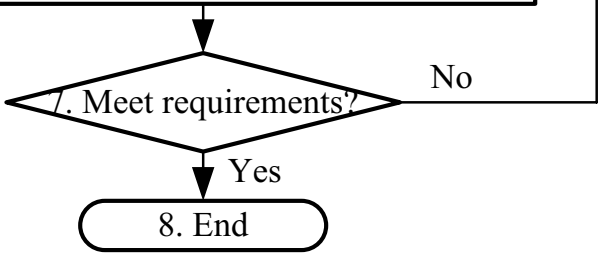

Figure 6 Flow chart for structural design procedure of metamorphic mechanism 


\section{Structural Design Illustrations of Metamorphic Mechanism with a Single Actuator}

Two examples are provided to verify the feasibility and practicality of the proposed design approach. Example one is a tablet press mechanism with a rotational actuator and two metamorphic prismatic joints. Example two is a repositioning mechanism that has a prismatic actuator and two metamorphic revolute joints.

\subsection{Example 1: Design of Metamorphic Mechanism for a Tablet Press Machine}

A tablet press machine is used to press powder into tablet products. The working process is described below, and illustrated in Figure 9. After a specific quantity of powder is discharged into the mold cavity, the upper punch moves to a prescribed position. Under the stress produced by the simultaneous squeeze operation between the upper and lower punches, the powder material is pressed into a tablet shape. In order to ensure processing quality, the simultaneous squeeze operation of the upper and lower punches must be held for a certain adjustable time, which means that the lower punch could remain static for a period of time, which can be conveniently adjusted according to different operational tasks.

\subsubsection{Source Mechanism Design of Tablet Press Machine}

According to the operational requirements, the output motion must consist of one prismatic (1P) movement at a minimum. In order to obtain the intermittent prismatic motion with an adjustable static time for the lower punch, two DOFs are required for the designed mechanism. The simplest structure composing a 2-DOF source mechanism for the metamorphic mechanism is one of the groups in Figure 4 connected to an actuator. Furthermore, in order to achieve 1P of the two output motions, only the RRRR is excluded from the nine groups in Figure 4 as the source mechanism. For this example, a PRRP group is connected to a rotary guide actuator and frame. Finally, the 2-DOF source mechanism for the tablet press metamorphic mechanism is designed as shown in Figure 7. Slider 4 is the lower punch in Figure 7, and thus, prismatic joint $P_{45}$ is clearly a metamorphic joint. Any

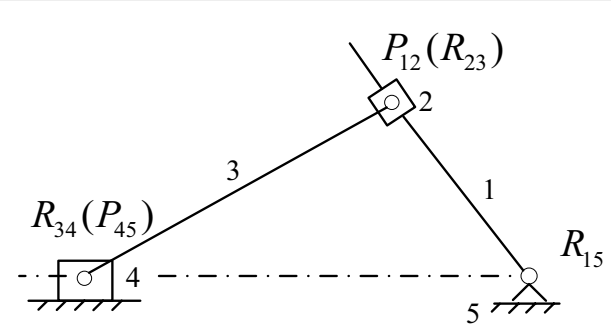

Figure 7 Source mechanism one of the joints $R_{34}, R_{23}$, or $P_{12}$ could be chosen as the last metamorphic joint. Selecting $P_{12}$ for this example to illustrate the design process, the two metamorphic joints $P_{12}$ and $P_{45}$ are determined.

The simultaneous operation of the upper punch and slider 4 (lower punch) should be maintained for a certain and adjustable time. The metamorphic mechanism to be designed should have two working configurations (stages). In working configuration I, slider 4 moves to the specified position and stops when it closely approaches the upper punch. In working configuration II, slider 2 moves along link 1 while slider 4 remains static. It should be noted that a useful feature of this design is that the length of time during which slider 4 and the upper punch remain in contact may be adjusted according to different powder materials. This may be accomplished simply through a change in the stiffness of the spring, as shown in the final design (Figure 11). Corresponding with the two working configurations, the metamorphic cyclogram of the tablet press metamorphic mechanism is obtained, as shown in Figure 8.

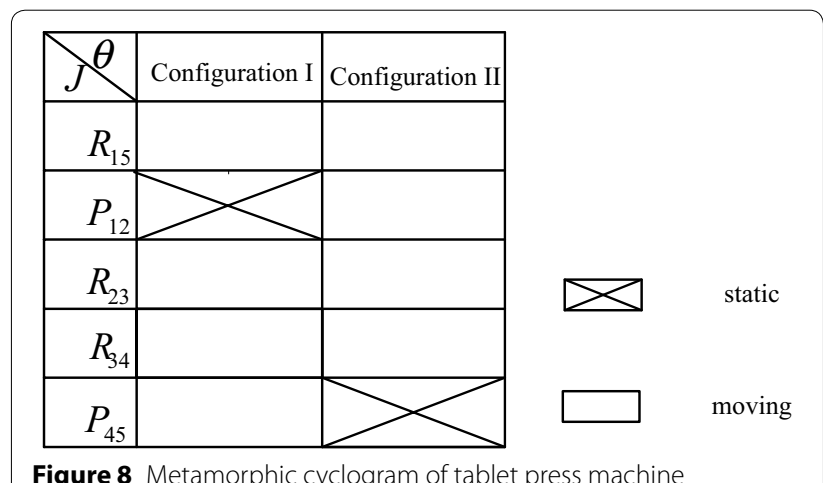

Figure 8 Metamorphic cyclogram of tablet press machine

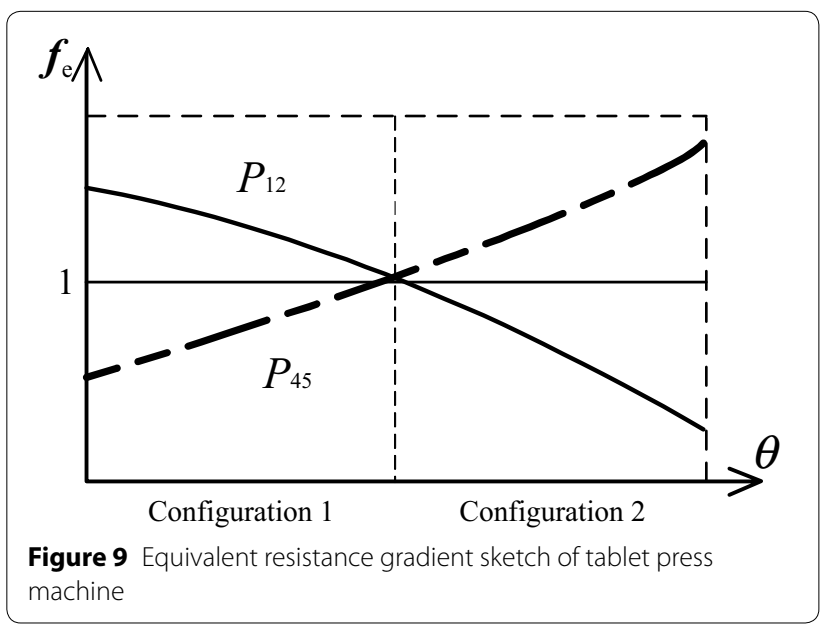




\subsubsection{Constraint Status Matrix of Metamorphic Joints}

Based on the metamorphic cyclogram and Eq. (2), the constraint status matrix of the metamorphic joints is deduced,

$$
\boldsymbol{W}=\left(\begin{array}{cc}
1_{12} & 0 \\
0 & 1_{45}
\end{array}\right)
$$

\subsubsection{Equivalent Resistance Matrix of Constrained Metamorphic Joints}

Based on the metamorphic cyclogram (Figure 5) and Eq. (3), the equivalent resistance gradient sketch for the tablet press machine is obtained, as shown in Figure 9.

According to the equivalent resistance gradient sketch, the equivalent resistance matrix is built based on Eq. (7):

$$
\boldsymbol{F}=\left(\begin{array}{ll}
0_{11} & f_{12} \\
f_{21} & 0_{22}
\end{array}\right)=\left(\begin{array}{cc}
0 & f_{45} \\
f_{12} & 0
\end{array}\right) .
$$

\subsubsection{Structural Synthesis Matrix of Metamorphic Mechanism}

According to the requirements of the kinematic/constraint status and equivalent resistance described above, the proper structures in the structural design catalogs of the metamorphic joints shown in Figure 3 are selected. Constraints that form vectors for the metamorphic joints $P_{12}$ and $P_{45}$ are solved based on Eqs. (9)-(11), that is $\boldsymbol{J}_{1}=(0 \mathrm{p} / \mathrm{pt} / \mathrm{ptg})$ and $\boldsymbol{J}_{2}=(p k / p t / p k g / p t g 0)$. Because $\boldsymbol{J}_{1}$ has three constraint forms and $\boldsymbol{J}_{2}$ has four constraint forms, it is easily calculated that the number of constrained forms $n$ of the metamorphic mechanism of the tablet press machine is $n=12$.

Finally, the structural synthesis matrix $C$ of the tablet press metamorphic mechanism is deduced according to Eq. (12), and $C_{i}(i=1-12)$ are obtained:

$$
\begin{array}{r}
\boldsymbol{C}=\left(\begin{array}{l}
\boldsymbol{J}_{1} \\
\boldsymbol{J}_{2}
\end{array}\right)=\left(\begin{array}{ll}
0_{11} & c_{12} \\
c_{21} & 0_{22}
\end{array}\right)=\left(\begin{array}{l}
\boldsymbol{P}_{45} \\
\boldsymbol{P}_{12}
\end{array}\right)=\left(\begin{array}{cc}
0 & c_{45} \\
c_{12} & 0
\end{array}\right) \\
=\left(\begin{array}{cc}
0 & p / p t / p t g \\
p k / p t / p k g / p t g & 0
\end{array}\right),
\end{array}
$$

$$
\begin{array}{lll}
C_{1}=\left(\begin{array}{cc}
0 & p \\
p k & 0
\end{array}\right), & C_{2}=\left(\begin{array}{cc}
0 & p \\
p t & 0
\end{array}\right), & C_{3}=\left(\begin{array}{cc}
0 & p \\
p k g & 0
\end{array}\right), \\
C_{4}=\left(\begin{array}{cc}
0 & p \\
p t g & 0
\end{array}\right), & C_{5}=\left(\begin{array}{cc}
0 & p t \\
p k & 0
\end{array}\right), & C_{6}=\left(\begin{array}{cc}
0 & p t \\
p t & 0
\end{array}\right), \\
C_{7}=\left(\begin{array}{cc}
0 & p t \\
p k g & 0
\end{array}\right), & C_{8}=\left(\begin{array}{cc}
0 & p t \\
p t g & 0
\end{array}\right), & C_{9}=\left(\begin{array}{cc}
0 & p t g \\
p k & 0
\end{array}\right), \\
C_{10}=\left(\begin{array}{cc}
0 & p t g \\
p t & 0
\end{array}\right), C_{11}=\left(\begin{array}{cc}
0 & p t g \\
p k g & 0
\end{array}\right), C_{12}=\left(\begin{array}{cc}
0 & p t g \\
p t g & 0
\end{array}\right) .
\end{array}
$$

There are 12 constraint forms of metamorphic joints in all, which are associated with 12 structures of constrained metamorphic mechanisms.

\subsubsection{Structural Diagrams of the Tablet Press Machine}

Corresponding to the structural synthesis matrix $C_{i}$ $(i=1-12)$, the 12 structural diagrams of tablet press metamorphic mechanisms are shown in Figure 10.

Considering the practical application, and according to the requirement of structural simplicity for reliability, a structural sketch map of the tablet press metamorphic mechanism corresponding to $\boldsymbol{C}_{3}$ is selected and designed as shown in Figure 11.

\subsection{Example 2: Design of Metamorphic Mechanism of Broken Strands Reposition Machine}

Broken strand repair of EHV power transmission lines is important for maintaining electrical grids. Compared with manual operation, developing a mobile robot repair/maintenance system is a safe and efficient way to solve the problem. Prior to the repair operation, broken strands should be repositioned to their original form. This could be accomplished by a broken strand repositioning component (such as the cut-open nut shown in Figure 12) with a special internal structure and motion mode [22]. To reduce the weight of the battery payload, a single-actuator metamorphic mechanism is designed to break the two cut-open nuts and lock the two cut-open nuts into a united nut, and then to accomplish broken strand reposition.

According to the operational requirements, two output motions are required. (1) The revolute (swing) output motion clamps the two cut-open nuts on the EHV power line. (2) The revolute output motion provides an actuator to lock the two cut-open nuts. According to the $2 R$ output motion, any one of RRRR, RRRP, RRPR, RRPP, RPRP, RPPR, and PRRP in Figure 4 could be chosen to construct the source mechanism. For this example, a 1-DOF group RRRR is selected to be connected to a slider actuator and frame, and a simple 2-DOF source mechanism is designed as shown in Figure 13.

According to the repositioning task, rocker 4 is fit to carry the cut-open nut in Figure 10(a) to clamp and tighten the EHV power line. Therefore, joint $D$ is a metamorphic joint. In order to obtain the other revolute output for locking the two cut-open nuts into a united nut as shown in Figure 10(b), any of the other revolute joints $A, B$, or $C$ could be chosen as the remaining metamorphic joint. Considering the practical operation, joint $C$ was selected as the other metamorphic joint. 

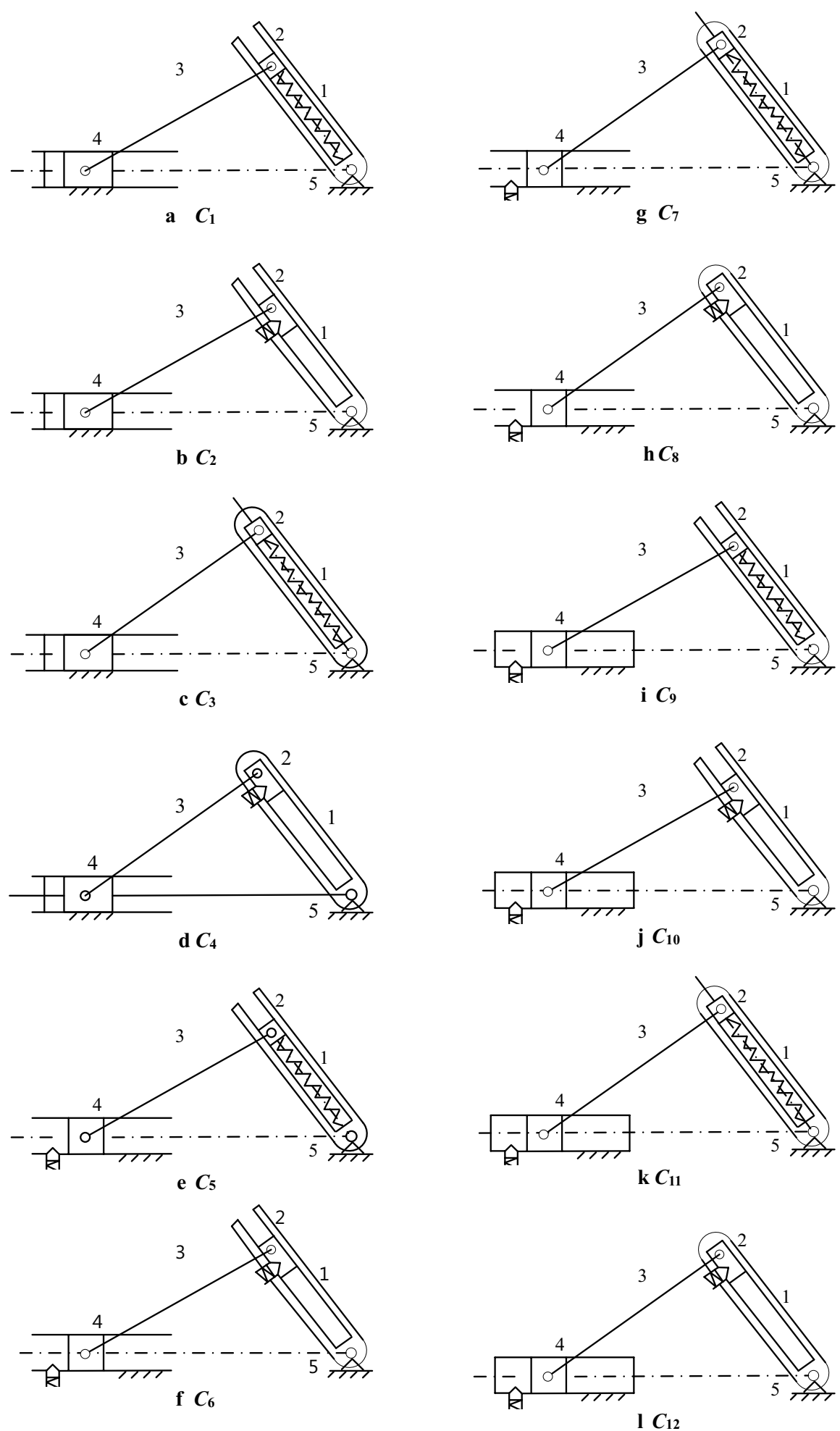

Figure 10 Structural diagrams of tablet press metamorphic mechanisms 

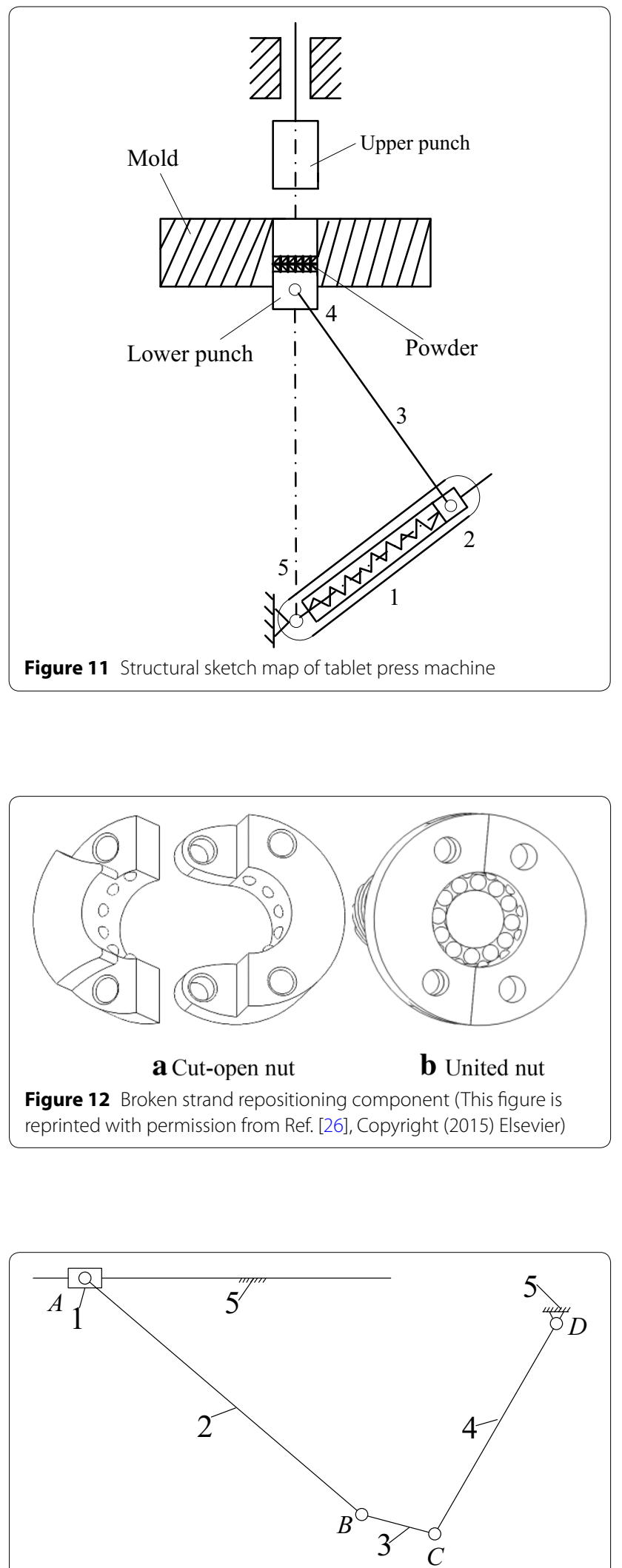

Figure 13 Source metamorphic mechanism for the repositioning machine

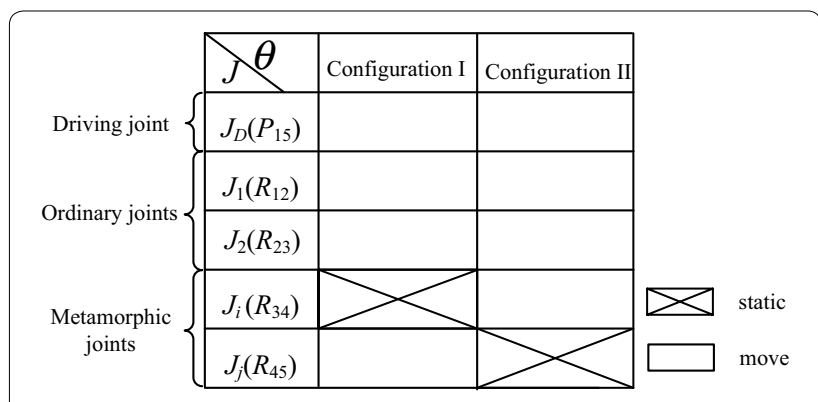

Figure 14 Metamorphic cyclogram of repositioning machine gradient sketch

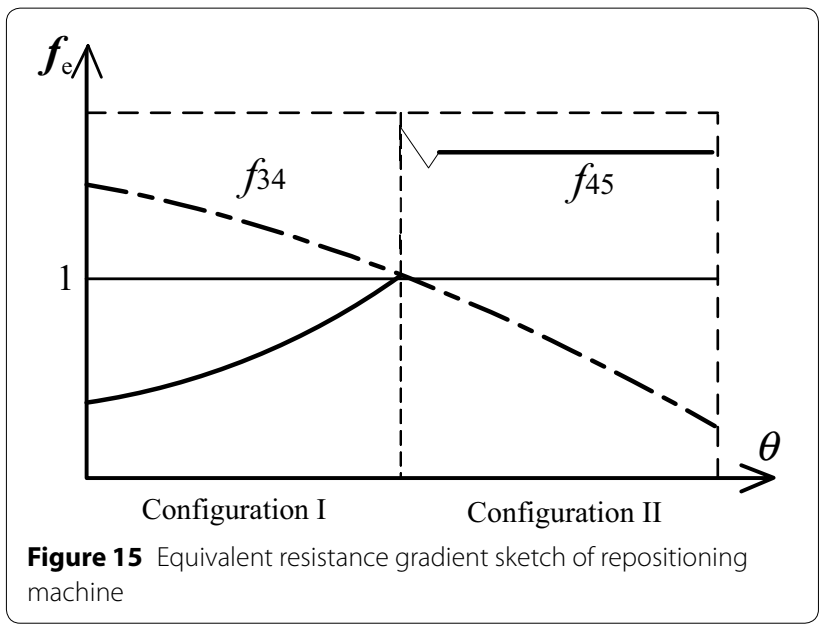

This metamorphic mechanism has two working configurations (stages). In working configuration I, rocker 4 , together with link 3 , swings to a specified position while metamorphic joint $C$ remains static and then stops when it reaches the power transmission line. In working configuration II, rocker 4 becomes fixed as a part of the frame because of the geometric constraint generated by the power line. Along with the continued motion of slider 1 , link 3 begins to rotate around joint $C$ after overcoming the resistance force of the metamorphic joint. Corresponding with the analysis of the two working configurations, the metamorphic cyclogram of the source mechanism is obtained, as shown in Figure 14, and the equivalent resistance gradient sketch for the broken strand repositioning machine is obtained as shown in Figure 15.

Based on Eqs. (9)-(11), the constraint form vectors of metamorphic joints $R_{45}$ and $R_{12}$ are found to be $J_{1}=(0 r / r t)$ and $\boldsymbol{J}_{2}=(r k / r t / r k g / r t g 0)$. Moreover, the structural synthesis matrix $C$ can be deduced from Eq. (12), and all eight structural matrices $C_{i}(i=1-8)$ are calculated from Eq. (15): 

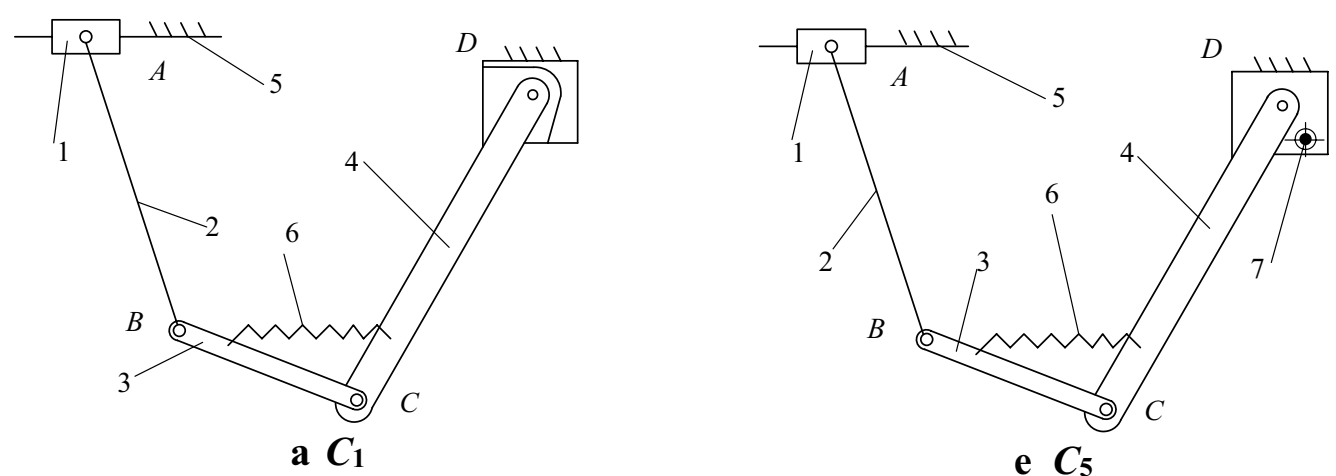

e $C_{5}$
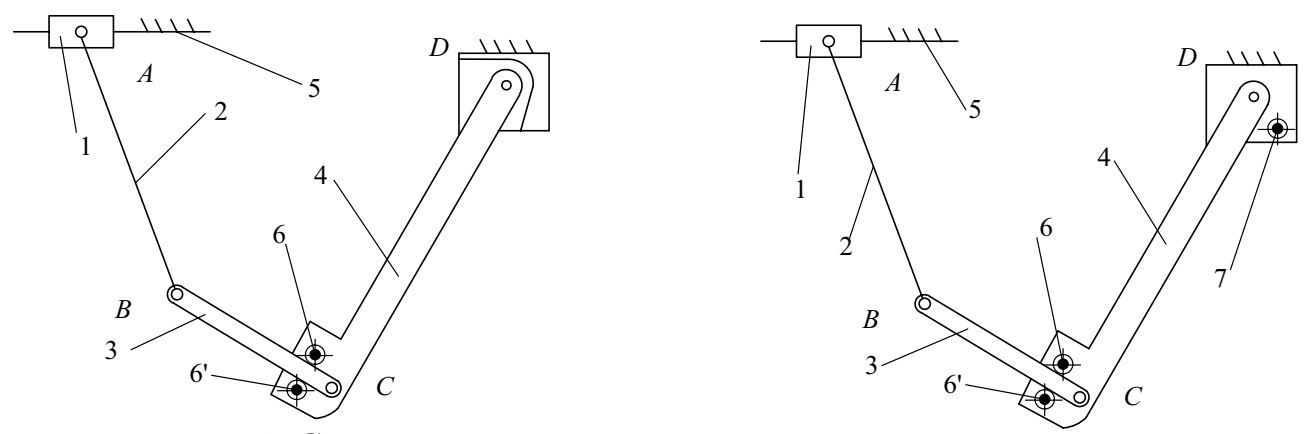

b $C_{2}$
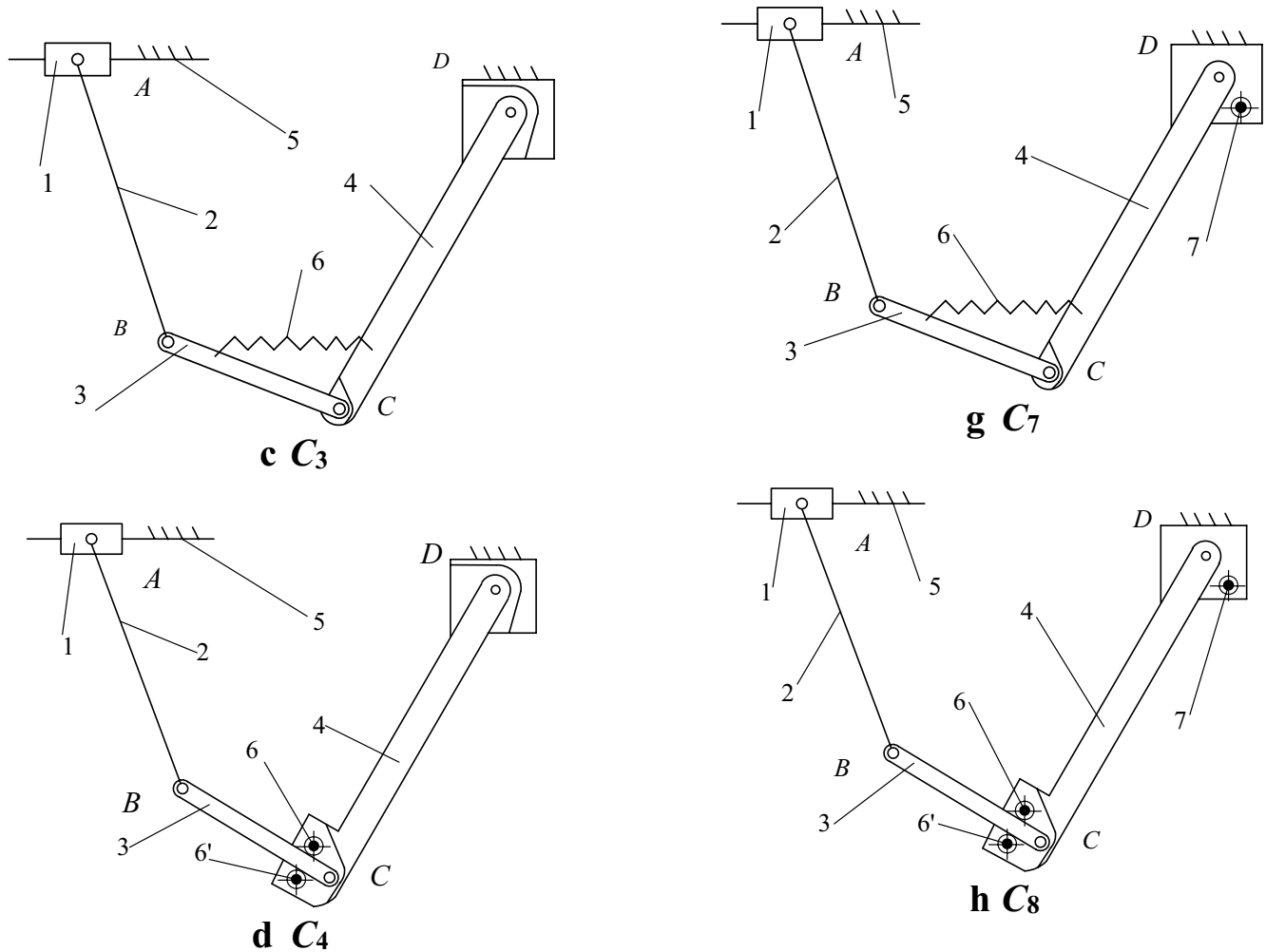

Figure 16 Eight structural diagrams of reposition metamorphic mechanisms 


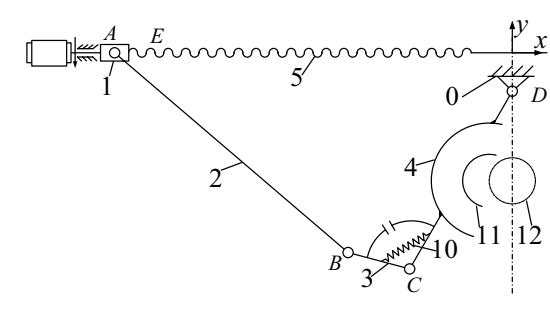

a Main two-mobility clamping metamorphic mechanism (configuration I)

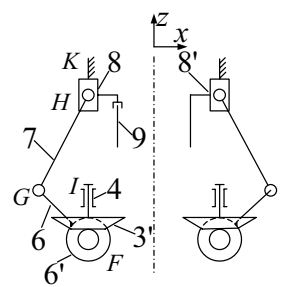

b Locking mechanism

Figure 17 Proposed broken strands reposition metamorphic mechanism

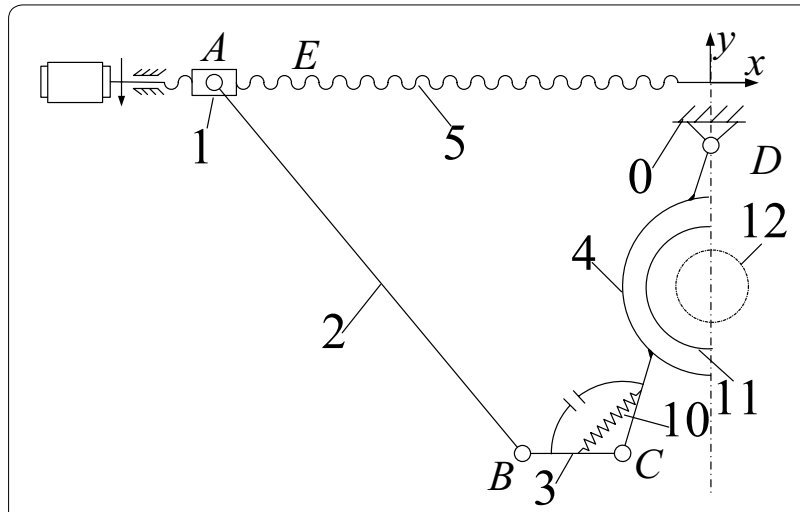

Figure 18 Configuration of changing work stage (configuration II)

$$
\begin{gathered}
C=\left(\begin{array}{l}
\boldsymbol{J}_{1} \\
\boldsymbol{J}_{2}
\end{array}\right)=\left(\begin{array}{ll}
0_{11} & c_{12} \\
c_{21} & 0_{22}
\end{array}\right)=\left(\begin{array}{c}
\boldsymbol{R}_{45} \\
\boldsymbol{R}_{34}
\end{array}\right) \\
=\left(\begin{array}{cc}
0 & c_{45} \\
c_{34} & 0
\end{array}\right)=\left(\begin{array}{cc}
0 & r / r t \\
r k / r t / r k g / r t g & 0
\end{array}\right) .
\end{gathered}
$$

There are eight structural synthesis matrices, implying that eight corresponding constrained metamorphic mechanisms can be theoretically designed to satisfy the broken strand repositioning operation. Finally, all eight structural diagrams for the repositioning metamorphic mechanisms are shown in Figure 16.

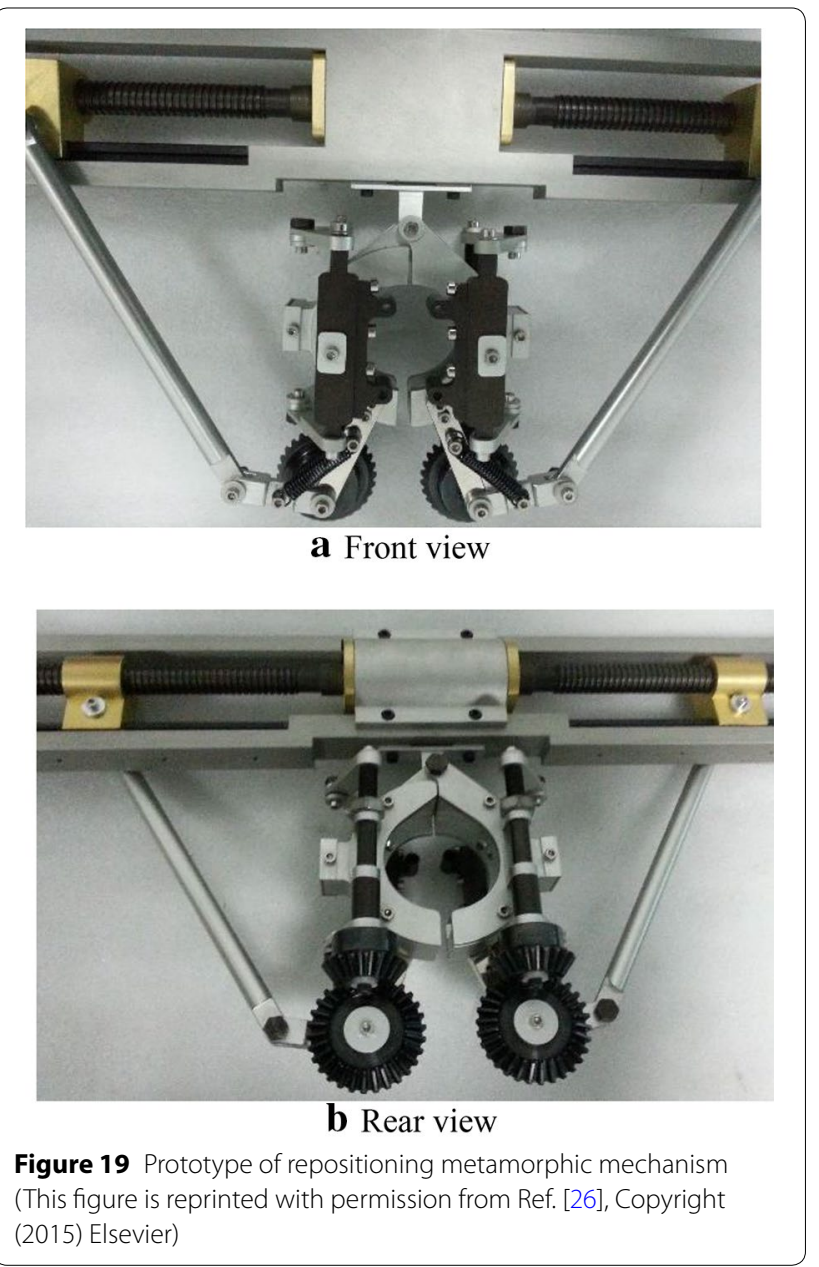

Considering the reliable working configuration transforming ability and structural simplicity, the mechanism in Figure 16(c) is selected as the final design and the practical broken strand repositioning metamorphic mechanism is designed as shown in Figure 17. (Considering the symmetrical operation of the practical mechanism, only half of the mechanism is shown in Figure 17(a) and Figure 18.) Metamorphic joint $C$ has a spring force constraint and a geometric constraint, and $D$ has a geometric constraint. Figure 17(a) shows the 2-DOF metamorphic mechanism, which makes two cut-open nuts 11 (shown in Figure 9) clamp on the EHV power line 12. As shown in Figure 17(b), the locking mechanism (a crank-slider mechanism) is used to unite the two cut-open nuts into one with pin 9. The driving motion of crank 6 is generated through a pair of bevel gears 3' and 6, which are rigidly connected to link 3 and crank 6 , respectively. Thus, the intermittent rotation of metamorphic joint $C$ (link 3 ) can drive the mechanism to lock the two cut-open nuts or disassemble the united nut after the repair operation. 
Figure 18 shows the changing transformation from working stage I to stage II. Figure 19 shows the prototype of the broken strand repositioning metamorphic mechanism for the EHV power transmission line.

\section{Discussion}

(1) A whole family of synthesizing approaches for source metamorphic mechanisms is not proposed in this paper. For instance, the structural syntheses based on 1-DOF groups of $n=5, p_{l}=7$ and $n=7$, $p_{l}=10$, or other pairs with more moving links are not discussed. In addition, 1-DOF groups of those containing general constraints, such as PPP are not considered to compose source mechanisms. Meanwhile, the synthesis approach proposed in this paper is only discussed for planar single-loop metamorphic mechanisms.

(2) With the continually enlarging design catalog describing metamorphic joint forms/structures [38, 39], more structural synthesis matrices and structural diagrams for metamorphic mechanisms will be obtained with different forms for metamorphic joints based on the same source mechanism. This will expand the practical application of constrained metamorphic mechanisms in the future.

\section{Conclusions}

(1) Considering the constraint forms/structures of metamorphic joints and the assembled combinations, a general structural design approach for a planar metamorphic mechanism was presented. According to the task-based metamorphic cyclogram, a constraint status matrix was proposed to describe the relationship between the working configuration transformation and variations of constrained forces. Then, a structural synthesis matrix was calculated by analyzing constraint forms, constraint forces, and the corresponding forms/structures of metamorphic joints. Finally, a structural design method based on the structural synthesis matrix was presented for designing a multi-DOF and multiconfiguration planar metamorphic mechanism with a single actuator.

(2) An effective design approach for composing source metamorphic mechanisms was proposed. Only 14 fundamental groups were chosen to make up thousands of source mechanisms for $n$-DOF planar single-loop metamorphic mechanisms. The simplest types of source mechanisms can be synthesized corresponding to the same task. In the future, traditional analysis methods based on Assur groups could be adopted to conveniently analyze the kinematics and forces of all the metamorphic mechanisms composed by the approach in this study.

\section{Acknowledgements}

Not applicable.

\section{Authors' Contributions}

QY was in charge of the whole research and wrote the initial manuscript; $\mathrm{GH}$ assisted with synthesis of the source metamorphic mechanism; SL assisted with structural design of metamorphic joints; HW was in charge of manufacture of prototype in Figure 19. HL revised the final manuscript. All authors read and approved the final manuscript.

\section{Authors' Information}

Qiang Yang, born in 1980, is currently an associate professor at School of Mechanical Engineering and Automation, Northeastern University, China. He received his doctor degree on mechanical engineering from Northeastern University, China, in 2009. His research interests include theory of mechanisms, metamorphic mechanisms, parallel mechanisms, and kinematic reliability of mechanisms. Tel: +86-24-83679346.

Guangbo Hao, born in 1981, is an associate professor at Department of Electrical and Electronic Engineering, University College Cork, Ireland. He received his doctor degree on mechanical engineering from Heriot-Watt University (HWU), UK, in 2011. His research interests include mechanisms and machine theory, design and application of complaint mechanisms, reconfigurable mechanisms.

Shujun Li is a professor at School of Mechanical Engineering and Automation, Northeastern University, China. His research interests include theory of mechanisms, theory of robot mechanisms, metamorphic mechanisms, and mechanical design theory and method.

Hongguang Wang is a professor at State Key Laboratory of Robotics, Shenyang Institute of Automation and Chinese Academy of Sciences. His research interests include the analysis and synthesis of robot mechanism, the mechanics of serial and parallel manipulators, the modular reconfigurable robots and autonomous mobile robots.

Haiyang Li is an associate professor at School of Mechanical Engineering and Automation, Northeastern University, China. His research interests include theory of mechanisms, complaint mechanisms.

\section{Funding}

Supported by National Natural Science Foundation of China (Grant No. 51575091, 51205052), Aeronautical Science Foundation of China (Grant No. 20170250001), and the Basic Science and Research Project of Chinese National University (Grant No. N160304008)

\section{Competing Interests}

The authors declare no competing financial interests.

\section{Author Details}

${ }^{1}$ College of Mechanical Engineering and Automation, Northeastern University, Shenyang 110819, China. ${ }^{2}$ Department of Electrical and Electronic Engineering, University College Cork, Cork T12 K8AF, Ireland. ${ }^{3}$ State Key Laboratory of Robotics, Shenyang Institute of Automation, Chinese Academy of Sciences, Shenyang 110016, China.

Received: 16 January 2020 Revised: 13 September 2020 Accepted: 15 October 2020

Published online: 02 November 2020 


\section{References}

[1] S Kota, A G Erdman. Motion control in production design. Mechanical Engineering, 1997, 119(8): 74-77.

[2] J S Dai, J J Rees. Mobility in metamorphic mechanisms of foldable/erectable kinds. Transactions of the ASME: Journal of Mechanical Design, 1999, 121(3): 375-382

[3] D L Li, J S Dai, Q X Zhang. Structure synthesis of metamorphic mechanisms based on the configuration transformations. Chinese J. Mech. Eng., 2002, 38(7): 12-16. (in Chinese).

[4] C H Liu, TL Yang. Essence and characteristics of metamorphic mechanisms and their metamorphic ways. The 11th World Congress in Mechanism and Machine Science, Tianjin, China, August 18-21, 2004: 1285-1288.

[5] J G Liu, Y C Wang, Bin Li, et al. Transformation technique research of the improved link-type shape shifting modular robot. Proceedings of the 2006 IEEE International Conference on Mechatronics and Automation, Luoyang, China, June 25-28, 2006: 295-300.

[6] D L Wang, J S Dai. Theoretical foundation of metamorphic mechanisms and its synthesis. Chinese J. Mech. Eng., 2007, 43(8): 32-42. (in Chinese)

[7] J S Dai, D L Wang. Geometric analysis and synthesis of the metamorphic robotic hand. Transactions of the ASME: Journal of Mechanical Design, 2007, 129(11): 1191-1196.

[8] Z H Lan, R Du. Representation of topological changes in metamorphic mechanisms with matrices of the same dimension. Transactions of the ASME: Journal of Mechanical Design, 2008, 130(7): 074501-1-4.

[9] X L Ding, Y Yang. Investigation of reconfiguration theory based on an assembly-circles artifact. ASME/IFTOMM International Conference on Reconfigurable Mechanisms and Robots, London, United Kingdom, June 22-26, 2009: 456-463.

[10] WX Zhang, X L Ding, J G Liu. A representation of the configurations and evolution of metamorphic mechanisms. Mechanical Sciences, 2016, 7(1): 39-47.

[11] J S Dai, J J Rees. Matrix representation of topological changes in metamorphic mechanisms. Transaction of ASME, Journal of Mechanical Design, 2005, 127(4): 837-840.

[12] S J LI, J S Dai. Augmented adjacency matrix for topological configuration of the metamorphic mechanisms. Journal of Advanced Mechanical Design, Systems, and Manufacturing, 2011, 5(5): 187-198.

[13] L P Zhang, D L Wang. Biological modeling and Evolution based synthesis of metamorphic mechanisms. Transactions of the ASME: Journal of Mechanical Design, 2008, 130(7): 072303-1-11.

[14] L P Zhang, J S Dai. Metamorphic techniques and geometric reconfiguration principles. ASME/IFTOMM International Conference on Reconfigurable Mechanisms and Robots, London, United Kingdom, June 22-26, 2009: 32-40.

[15] Z H Lan. Structure and evolution of planar metamorphic mechanisms. Conference on Applied Mechanisms and Machine Science, Shanghai, China, July, 21-25, 2010: 104-106.

[16] KT Zhang, J S Dai, Y F Fang. Topology and constraint analysis of phase change in the metamorphic chain and its evolved mechanism. Transactions of the ASME: Journal of Mechanical Design, 2010, 132(12): 121001-1-11.

[17] F Yang, J G Tao, Z Q Deng. New method to describe the structure changing of metamorphic mechanisms and its application in structure synthesis. Journal of Mechanical Engineering, 2011, 47(15): 1-8. (in Chinese)

[18] D M GAN, J S Dai, J Dias, et al. Constraint-plane-based synthesis and topology variation of a class of metamorphic parallel mechanisms. Journal of Mechanical Science and Technology, 2014, 28(10): 4179-4191.

[19] D M Gan, J S Dai, Q Z Liao. Constraint analysis on mobility change of a novel metamorphic parallel mechanism. Mechanism and Machine Theory, 2010, 45(8): 1864-1876.

[20] W X Zhang, X L Ding, J S Dai. Morphological synthesis of metamorphic mechanisms based on constraint variation. Journal of Mechanical Engineering Science, 2011, 225(12): 2297-2310.

[21] C Valsamos, V Moulianitis, N Aspragathos. Kinematic synthesis of structures for metamorphic serial manipulators. Transactions of the ASME: Journal of Mechanical Design, 2014, 6(11): 041005-1-14.
[22] Q Yang, H G Wang, S J Li. Structural synthesis for broken strands repair operation metamorphic mechanism of EHV transmission lines. Journal of Vibroengineering, 2014, 16(6): 2768-2778

[23] XW Kong, M Pfurner. Type synthesis and reconfiguration analysis of a class of variable-DOF single-loop mechanisms. Mechanism and Machine Theory, 2015, 85(3): 116-128.

[24] XW Kong, J J Yu. Type synthesis of two-degrees-of-freedom 3-4R parallel mechanisms with both spherical translation mode and sphere-on-sphere rolling mode. Transactions of ASME: Journal of Mechanisms and Robotics, 2015, 7(4): 041018-1-8.

[25] S J Li, J S Dai. Structure synthesis of single-driven metamorphic mechanisms based on the augmented Assur groups. Transactions of ASME: Journal of Mechanisms and Robotics, 2012, 4(3): 031004-1-8.

[26] S J Li, H G Wang, Q L Meng, et al. Task-based structure synthesis of source metamorphic mechanisms and constrained forms of metamorphic joints. Mechanism and Machine Theory, 2016, 96(8): 334-345.

[27] S J Li, H G Wang, J S Dai. Assur-group inferred structural synthesis for planar mechanisms. Transactions of ASME, Journal of Mechanisms and Robotics, 2015, 7(4): 041001-1-9.

[28] S J Li, J S Dai. The equivalent resistance gradient model of metamorphic mechanisms and the design approach. In: J S Dai, M Zoppi, X Kong (eds). Advances in reconfigurable mechanisms and robotics I, Springer Press, 2012 : 53-62.

[29] H B Tian, HW Ma, K Ma. Method for configuration synthesis of metamorphic mechanisms based on functional analyses. Mechanism and Machine Theory, 2018, 123(2): 27-39.

[30] C Zhao, H W Guo, Y Q Liu, et al. Design and kinematic analysis of a 3RRIS metamorphic parallel mechanism for large-scale reconfigurable space multifingered hand. Transactions of ASME: Journal of Mechanisms and Robotics, 2018, 10(8): 041012-1-12.

[31] J Wei, J S Dai. Reconfiguration-aimed and manifold-operation based type synthesis of metamorphic parallel mechanisms with motion between 1R2T and 2R1T. Mechanism and Machine Theory, 2019, 139(9): 66-80.

[32] J Wei, J S Dai. Lie group based type synthesis using transformation configuration space for reconfigurable parallel mechanisms with bifurcation between spherical motion and planar motion. Transactions of ASME: Journal of Mechanical Design, 2020, 142(6): 063302-1-13.

[33] XW Kong, C M Gosselin, P L Richard. Type synthesis of parallel mechanisms with multiple operation modes. Transactions of ASME: Journal of Mechanical Design, 2007, 129(6): 595-601.

[34] J Angeles. The qualitative synthesis of parallel manipulators. Transactions of ASME: Journal of Mechanical Design, 2004, 126(4): 617-624.

[35] XW Kong. Type synthesis of 3-DOF parallel manipulators with both a planar operation mode and a spatial translational operation mode. Transactions of ASME, Journal of Mechanisms and Robotics, 2013, 5(4): 041015-1-8.

[36] D L Li, Z H Zhang, G M Chen. Configuration analysis of metamorphic mechanisms based on extended adjacency matrix operations. Chinese Journal of Mechanical Engineering, 2011, 24(5): 767-773.

[37] D L Li, Z H Zhang, G M Chen. structural synthesis of compliant metamorphic mechanisms based on adjacency matrix operations. Chinese Journal of Mechanical Engineering, 2011, 24(4): 522-528.

[38] H S Yan, C H Kuo. Topological representations and characteristics of variable kinematic joints. Transaction of ASME: Journal of Mechanical Design, 2006, 128(3): 384-391.

[39] J N Liu, W B Zhang. Type synthesis of metamorphic mechanism based on structure realization of metamorphic joint. Journal of South China University of Technology, 2018, 45(9): 19-25. (in Chinese). 